\title{
Metaanalyse: Übergewicht erhöht Komplikationen bei Hüft-TEP
}

Onggo JR et al. Greater risk of complications, infections, and revisions in the obese versus non-obese total hip arthroplasty population of 2,190,824 patients: a meta-analysis and systemic review. Osteoarthritis Cartilage 2020; 28: $31-44$

Eine aktuelle Metaanalyse vergleicht Outcomes, Komplikationen und perioperative Parameter beim Einsatz von Totalendoprothesen (TEP) der Hüfte bei adipösen Patienten (BMI $\geq 30 \mathrm{~kg} / \mathrm{m}^{2}$ ) und nicht-adipösen Patienten (BMI $\leq$ $30 \mathrm{~kg} / \mathrm{m}^{2}$ ). Auch wurde eine Subgruppenanalyse mit krankhaft fettleibigen Patienten (BMI $\geq 40 \mathrm{~kg} / \mathrm{m}^{2}$ ) durchgeführt.

Für die Metaanalyse suchten die Autoren in verschiedenen Datenbanken (PubMed, Medline und EMBASE) nach Studien jeglichen Studientyps, in denen Outcomes und Komplikationen nach Hüft-TEP bei adipösen und nicht-adipösen Patienten verglichen wurden. Die Autoren hielten sich bei der Suche an die PRISMA Kriterien. In die Auswertung schlossen die Wissenschaftler schließlich 67 englischsprachige Studien ein, darunter 19 prospektive, 39 retrospektive und 9 Fall-Kontroll-Studien. 
In die Analyse gingen Daten von insgesamt 581012 adipösen und 1609812 nicht-adipösen Patienten ein. In 47 Studien war eine Geschlechterverteilung angegeben, $61 \%$ der übergewichtigen Probanden in diesen Studien waren weiblich und 60,7\% der nicht-übergewichtigen Probanden. In 54 Studien wurde das Alter der Probanden bei der Hüft-TEP angegeben, in der Gruppe der Übergewichtigen lag das mittlere Alter zwischen 50 und 74,5 Jahren, in der Gruppe der nicht-Übergewichtigen zwischen 48 und 77,2 Jahren.

Die Metaanalyse ergab, dass adipöse Patienten ein erhöhtes Risiko für alle Komplikationen (OR=1,53, 95\% Kl: 1,30-1,80, $p<0,001)$, tiefe Infektionen (OR=2,71, $95 \% \mathrm{KI}: 2,08-$ $3,53, p<0,001)$, oberflächliche Infektionen (OR=1,99, 95\% KI: 1,55-2,55, $\mathrm{p}<0,001)$ Dislokationen $(\mathrm{OR}=1,72,95 \% \mathrm{KI}: 1,66-1,79$, $\mathrm{p}<0,001)$, Re-Operationen ( $O R=1,61,95 \%$ $\mathrm{Kl}: 1,40-1,85, \mathrm{p}<0,001)$, Revisionen (OR= $1,44,95 \% \mathrm{Kl}: 1,32-1,57, \mathrm{p}<0,001)$ und Wiederaufnahmen $(\mathrm{OR}=1,37,95 \% \mathrm{KI}: 1,15-$ $1,63, p<0,001)$ hatten.

Allerding hatten die übergewichtigen Patienten kein erhöhtes Risiko für venöse Thromboembolien $(\mathrm{OR}=1,44,95 \% \mathrm{KI}$ : $0,93-2.21, p=0,10)$, peri-prothetische Frakturen $(\mathrm{OR}=1,12,95 \% \mathrm{KI}: 0,98-1,28$, $\mathrm{P}=0,08)$, aseptische Lockerungen ( $O R=$ $0,98,95 \% \mathrm{KI}: 0,78-1,22, \mathrm{p}=0,84)$ oder Nervenparesen (OR $=0,90,95 \% \mathrm{KI}: 0,30-$ $2,67, p=0,84)$.

Die Subgruppenanalyse ergab, dass die Risiken für Komplikationen bei krankhaft fettleibigen Patienten $(n=85613)$ mit einem $\mathrm{BMI} \geq 40 \mathrm{~kg} / \mathrm{m}^{2} \mathrm{im}$ Vergleich zu den nichtübergewichtigen Patienten $(n=1123803)$ noch größer sind: für alle Komplikationen $(\mathrm{OR}=2,68,95 \% \mathrm{Kl}: 2,03-3,53, \mathrm{p}<0,001)$, tiefe Infektionen (OR = 3,69, $95 \% \mathrm{KI}: 3,16$ $4,30, p<0,001)$, oberflächliche Infektionen (OR=4,95, $95 \% \mathrm{KI}: 3,87-6,33, \mathrm{p}<0,001)$, Re-Operationen (OR=2,96, $95 \% \mathrm{KI}: 2,21$ 3,96, $p<0,001)$, Revisionen (OR=2,17, 95\% Kl: 1,90-2,48, $p<0,001)$, Dislokationen (OR=2,12, $95 \% \mathrm{KI}: 2,03-3,53, \mathrm{p}<0,001)$ und Wiederaufnahmen $(O R=1,99,95 \% \mathrm{Kl}$ : 1,81-2,20, p<0,001). Die Risiken für venöse Thromboembolien, peri-prothetische Frakturen und aseptische Lockerungen waren im Gegensatz zu den nicht-Übergewichtigen Probanden nicht erhöht. 
Die Wissenschaftler konnten außerdem zeigen, dass übergewichtige Patienten eine signifikant höhere mittlere Operationsdauer hatten (Mittlere Differenz 8,71 Minuten) und länger im Krankenhaus verbleiben mussten (Mittlere Differenz 0,45 Tage). Aufgrund der unterschiedlichen Messmethoden in den einzelnen Studien konnten in der Metaanalyse keine Patienten-berichteten Outcomes verglichen werden. Auch konnten keine Überlebensanalysen durchgeführt werden.

\section{FAZIT}

Die vorliegende Metaanalyse zeigt, dass Übergewicht das Risiko für Komplikationen bei der Hüft-TEP stark erhöht. Die Autoren empfehlen, übergewichtige Patienten besonders über mögliche Komplikationen aufzuklären. Auch solle bei diesen Patienten besonders auf das Auftreten von Komplikationen geachtet werden.

Marisa Kurz M. Sc. B. A., München 